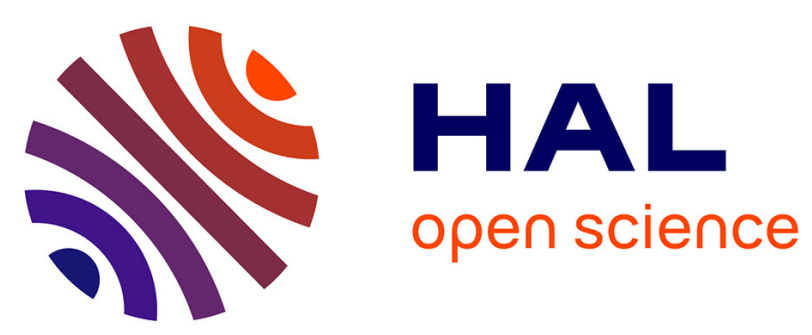

\title{
CONTRÔLE DE LA CUISSON DE THERMODURCISSABLES PAR IMPLANT PIÉZOÉLECTRIQUE : ÉTUDES PRATIQUE ET THÉORIQUE
}

I. Perrissin-Fabert, Y. Jayet, G. Merle

\section{To cite this version:}

I. Perrissin-Fabert, Y. Jayet, G. Merle. CONTRÔLE DE LA CUISSON DE THERMODURCISSABLES PAR IMPLANT PIÉZOÉLECTRIQUE: ÉTUDES PRATIQUE ET THÉORIQUE. Journal de Physique IV Proceedings, 1992, 02 (C1), pp.C1-887-C1-890. 10.1051/jp4:19921193 ～jpa-00251158

\author{
HAL Id: jpa-00251158 \\ https://hal.science/jpa-00251158
}

Submitted on 1 Jan 1992

HAL is a multi-disciplinary open access archive for the deposit and dissemination of scientific research documents, whether they are published or not. The documents may come from teaching and research institutions in France or abroad, or from public or private research centers.
L'archive ouverte pluridisciplinaire HAL, est destinée au dépôt et à la diffusion de documents scientifiques de niveau recherche, publiés ou non, émanant des établissements d'enseignement et de recherche français ou étrangers, des laboratoires publics ou privés. 


\title{
CONTRÔLE DE LA CUISSON DE THERMODURCISSABLES PAR IMPLANT PIEZOELLCTRIQUE : ÉTUDES PRATIQUE ET THÉORIQUE
}

\author{
I. PERRISSIN-FABERT, Y. JAYET et G. MERLE* \\ Laboratoire du Traitement du Signal et d'Ultrasons, Bàtiment 502, I.N.S.A., 20 avenue Albert \\ Einstein, F-69621 Villeurbanne cedex, France \\ *Laboratoire des Matériaux Macromoléculaires, L.N.S.A., 20 avenue Albert Einstein, \\ F-69621 Villeurbanne cedex, France
}

\begin{abstract}
An original ultrasonic method for monitoring the curing of polymers is described here. This method is based on the study of the deformations of a thin piezoelectric disc excited electrically and inserted inside the medium to control. A theoretical study is here developped and its valididity is shown by confronting the theoretical and experimental results.
\end{abstract}

\section{RESUME}

Nous présentons une nouvelle méthode ultrasonore qui permet le contrôle de l'état de cuisson d'une structure polymère. Cette méthode est basée sur l'étude des déformations d'un disque piézoélectrique excité électriquement et inséré dans le matériau à contrôler. Une étude théorique est ici développée et sa validité est montrée par confrontation des résultats théoriques et expérimentaux.

\section{INTRODUCTION}

Lorsqu'un disque piézoélectrique est soumis à une excitation électrique, il se déforme selon son rayon (mode radial) et selon son épaisseur (mode axial) et la valeur de son impédance électrique est fonction de l'amplitude de sa déformation. Ces deux modes de déformation sont caractérisés par une fréquence et par une amplitude de déformation propres. Lorsque l'épaisseur de l'élément est très petite devant son rayon, ses modes de vibration axial et radial sont situés dans des domaines de fréquence très éloignés si bien qu'il est possible de les étudier séparément. Dans ces conditions les vibrations axiales de l'élément actif peuvent être simulées à l'aide d'un modèle unidimensionnel (la modélisation du mode de vibration radiale est plus complexe, nous ne développerons ici pas son expression). L'expression analytique de l'impédance électrique axiale alors obtenue s'exprime en fonction des caractéristiques piézoélectrique et mécanique de la céramique et des propriétés viscoélastiques du milieu qui entoure l'élément actif. La validité du modèle établi est montré par confrontation des impédances électriques mesurée expérimentalement et calculée à partir du modèle, dans le cas où la céramique est placée dans l'air, dans un liquide et dans un solide.

La dernière partie de cette étude est consacrée à la description de la méthode développée pour suivre la polymérisation de résine thermodurcissable. La validité de cette méthode est montrée par confrontation des résultats obtenus avec ceux issus d'une étude par méthode d'échographie ultrasonore.

\section{MODELISATION DU MODE DE VIBRATION AXIAL DE LA CERAMIQUE.}

L'étude pratique des vibrations de la céramique étant effectuée dans le domaine de Laplace, l'étude théorique développée est menée directement dans le domaine fréquentiel. Si on considère que l'épaisseur de la céramique est très petite devant son rayon, il est possible de simuler le mode de vibration axial de la 
céramique à l'aide d'un modèle unidimensionnel. Dans cette hypothese en effet, les différentes grandeurs vectorielles sont symétriques par rapport à n'importe quel axe zz' perpendiculaire à la surface plane du disque et donc seule leur composante définies selon l'axe zz' est non nulle. Le modèle utilisé pour cette étude est présenté sur la figure 1.

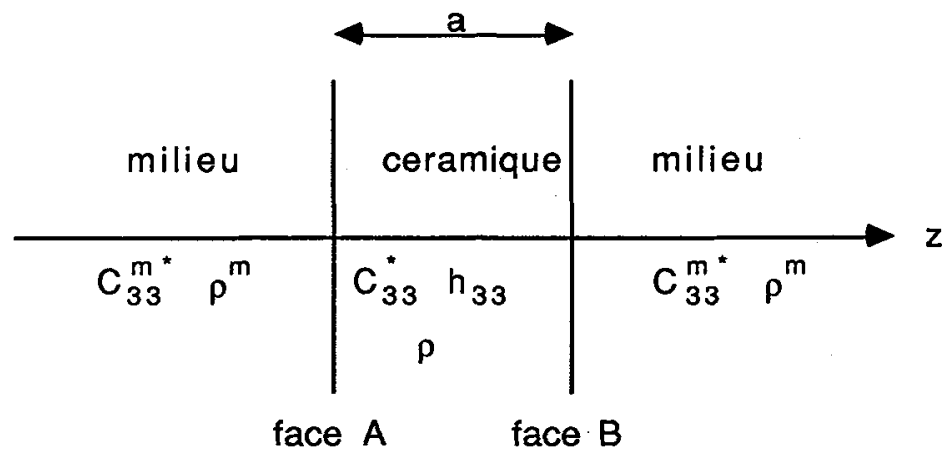

Figure 1: Modélisation du mode de vibration axial de la céramique.

Sur cette figure, h33 est le coefficient piézoélectrique de la céramique, et $\rho$ la densité et $\mathrm{C}_{33}{ }^{*}$ le coefficient viscoélastique défini par: $\mathbf{C}_{33}{ }^{*}=C_{33}+p \eta \mathbf{3 3}, C_{33}$ étant la constante d'élasticité du milieu considéré et $\eta 33$ sa viscosité.

Dans ces conditions, l'expression de la tension électrique présente aux bornes de l'élément actif est donnée par: $V_{\mathbf{a}}(p)=\int_{0}^{a} \mathbf{E}(z, p) d z$, où a représente l'épaisseur de l'élément actif. L'expression du champ éléctrique $\mathrm{E}(\mathrm{z}, \mathrm{p})$ est donnée par les équations générales de la piézoélectricité et s'écrit:

$$
E(z, p)=-h_{33} \frac{\partial U(z, p)}{\partial z}+\beta 33 D(z, p),
$$

où $\beta 33$ est la composante selon l'axe $z z^{\prime}$ de l'impermittivité de la céramique.

Par ailleurs, le théorème de Gauss montre que dans un milieu piézoélectrique et en dehors des charges divD=0, ce qui signifie que: $D(z, p)=\frac{1}{S_{e}} \frac{I(p)}{p}$ où $I(p)$ est le courant appliqué aux bornes de la céramique.

Ainsi donc: $V_{\mathbf{a}}(\mathbf{p})=-\mathbf{h}_{33}\{\mathrm{U}(\mathbf{a}, \mathbf{p})-\mathrm{U}(0, \mathrm{p})\}+\beta 33 \frac{\mathbf{a}}{\mathbf{S}_{\mathbf{e}}} \frac{\mathbf{I}(\mathbf{p})}{\mathbf{p}}$.

L'expression du déplacement $U(z, p)$ des faces $A$ et $B$, est obtenue à partir des équations différentielles qui expriment la propagation des ondes ultrasonores dans chacun des milieux. Elles sont de la forme: $p^{2} U(z, p)=C_{33} * \frac{\partial U(z, p)}{\partial z^{2}}$. La solution de cette équation est du type $U(x, p)=K_{1} \exp (\lambda z)+K_{2} \exp (\lambda z)$, où $K_{1}$ et $K_{2}$ sont les constantes d'intégration calculées en tenant compte des conditions aux limites qui impliquent qu'il y ait continuité des composantes normales des contraintes et des déplacements à la périphérie de l'élément actif. Dans de telles conditions l'expression de la tension présente aux bornes de l'élément actif se développe sous la forme:

$$
\begin{aligned}
V_{a}(p)= & \frac{-h_{33}{ }^{2} I(p)}{\left(Z_{m}{ }^{*}+Z^{*}\right) S_{e p} p^{2}}\left\{\frac{\left(1-R_{m}{ }^{*} \exp (-2 \lambda a)-\left(1-R_{m}{ }^{*}\right) \exp (-2 \lambda a)\right)^{2}}{\left(1-R_{m}{ }^{*} \exp (-2 \lambda a)\right)\left(1-R_{m}{ }^{*} \exp (-2 \lambda a)\right)}\right. \\
& \left.+\frac{(1-\exp (-2 \lambda a))}{\left(1-R_{m}{ }^{*} \exp (-2 \lambda a)\right)}\right\}+\frac{\beta 33 a(p)}{S_{e p}}
\end{aligned}
$$


Où $\mathrm{Z}^{*}$ et $\mathrm{Z}_{\mathrm{m}}$ * sont les impédances acoustiques complexes de la céramique et du milieu qui l'entoure. $\mathbf{R}_{\mathbf{m}}{ }^{*}=\frac{\mathbf{Z}_{\mathbf{m}}{ }^{*}-\mathbf{Z}^{*}}{\mathbf{Z}_{\mathbf{m}}{ }^{*}+\mathbf{Z}^{*}}$ est le coefficient de réflection complexe défini à l'interface céramique-milieu. $\lambda^{*}=\frac{\mathbf{p} \mathbf{a}}{\mathbf{C}^{\prime *}}$ est défini par le produit du nombre d'onde complexe et de l'épaisseur a de la céramique et $S_{\mathrm{e}}$ est la surface de l'élément actif.

La valeur de la tension présente aux bornes de la céramique dépend des propriétés viscoélastiques du milieu qui l'entoure par l'intermédiaire du coefficient de réflection. L'impédance électrique axiale de la céramique définie par $\mathbf{Z}_{\mathbf{a}}{ }^{*}=\frac{V_{\mathbf{a}}(\mathbf{p})}{\mathbf{I}(\mathbf{p})}$, est donc liée aux composantes selon l'axe $\mathrm{zz}^{\prime}$ des caractéristiques élastique et visqueuse du milieu qui entoure l'élément actif.

L'impédance électrique axiale obtenue par simulation numérique (Figure 2) est en bon accord avec celle mesurée expérimentalement (Figure 3), que le milieu qui entoure la céramique soit de l'air, un liquide ou un solide.

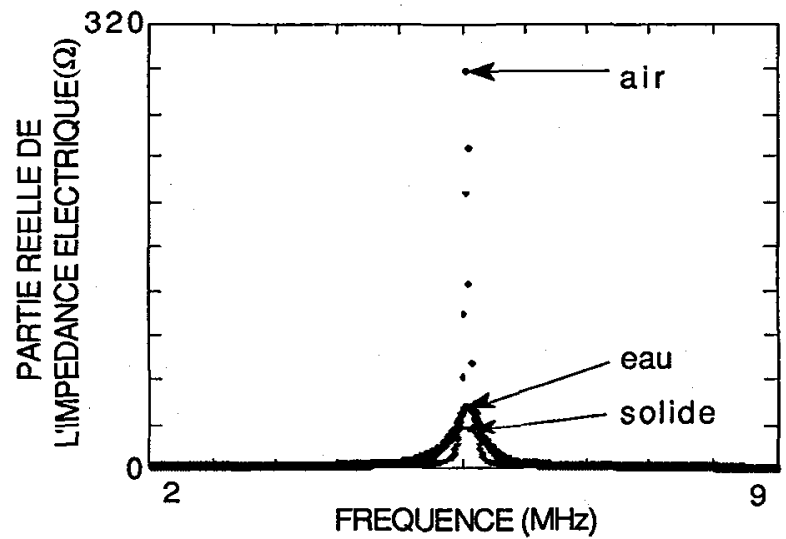

Figure 2: Impédance électrique théorique de la cémique placée dans l'air, dans un liquide et dans un solide

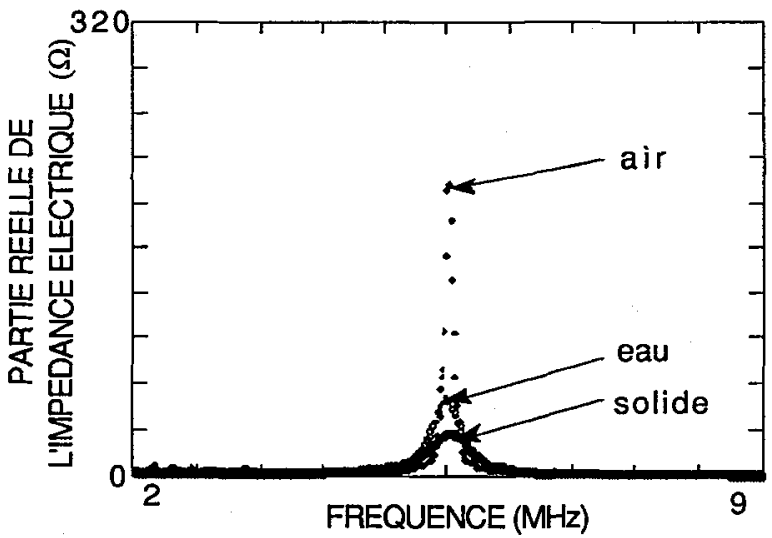

Figure 3: Impédance électrique expérimentale de la cémique placée dans l'air, dans un liquide et dans un solide

\section{METHODE DEVELOPPEE POUR SUIVRE LE DURCISSEMENT D'UNE RESINE.}

Les courbes précédentes montrent que l'amortissement des vibrations de la céramique est fonction des propriétés viscoélastiques du milieu qui entoure l'élément piézoélectrique. Cet amortissement se traduit sur 
le spectre de l'impédance électrique de la céramique par une diminution de l'amplitude des différents pics de vibration et par une augmentation de leur largeur de bande. L'évolution de ces deux paramètres est alors d'autant plus importante que les propriétés viscoélastiques du milieu sont proches de celles de la céramique. En étudiant leur variation il est donc possible de suivre la transformation du milieu dans lequel est placé l'élément actif de l'état liquide à l'état solide (ex: vitrification d'une résine).

Nous avons ainsi étudié le durcissement d'une résine époxyde et comparé les résultats que nous obtenons à ceux issus d'une étude par méthode d'échographie ultrasonore.

Le facteur principal qui régit la vitrification d'une resine est la diminution de la mobilité des segments de chaine macromoléculaire. Cette transition est définie sur un certain domaine temporel qui est fonction à la fois de la nature chimique de la résine et de la méthode d'investigation: plus la fréquence de sollicitation de la résine est grande et plus le phénomène est détecté rapidement. La fréquence de vibration de l'élément actif utilisé étant de $5 \mathrm{MHz}$ nous avons utilisé un traducteur large bande de fréquence nomminale $5 \mathrm{MHz}$ pour générer des ondes ultrasonores de type longitudinale dans le milieu de propagation.

Sur la figure 4 sont présentées l'évolution du paramètre "amplitude + largeur de bande relative" obtenue par notre nouvelle méthode (courbe 1) et les variations relatives de la vitesse de propagation (courbe 2) et de l'atténuation des ondes ultrasonores (courbe 3) dans la résine en fonction du temps de polymérisation. Initialement, la vitrification de la résine se traduit par une diminution du paramètre "amplitude+largeur de bande relatives" en conséquence de l'évolution du couplage mécanique entre la céramique et la résine ${ }^{1}$, puis lorsque la résine devient élastique, par une augmentation de ce paramètre ${ }^{1}$. La vitesse de propagation des ondes ultrasonores dans la résine évolue à partir d'un temps $T$ analogue à celui pour lequel est obtenu le minimum de la courbe (1). Son évolution est ensuite proportionnelle à la variation de la constante d'élasticité C33 de la résine. L'atténuation des ultrasons dans le milieu de propagation varie quant à elle, en fonction de la viscosité du mélange réactionnel. Elle passe par un maximum au temps $T$, puis diminue lorsque le matériau devient vitreux.

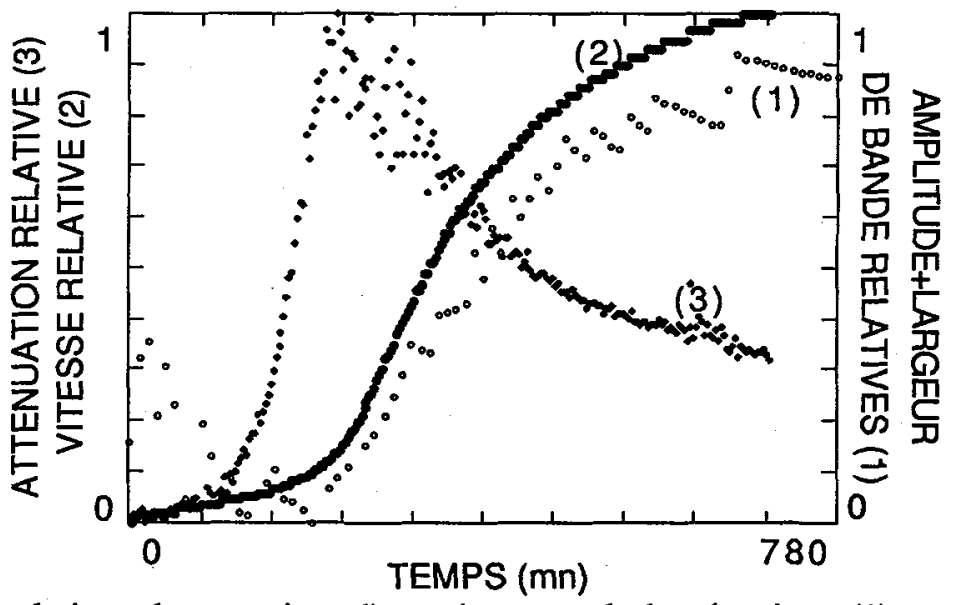

Figure 4: Evolutions du paramètre d'amortissement de la céramique (1) et de la vitesse de propagation (2) et de l'atténuation des ultrasons observées lors du durcissement de la résine.

La bonne concordance de l'évolution des enregistrements montre la validité de la méthode établie. Cette méthode permet de suivre la cinétique de la réaction qui se déroule dans le milieu qui entoure l'élément actif et peut-être utilisée pour contrôler son degré d'avancement. Il faut toutefois ajouter qu'une étude plus complète de la polymérisation de la résine est permise par l'exploitation selon le même procédé des harmoniques de vibration radiale de la céramiquel.

\section{REFERENCE.}

1-Monitoring the thermosets polymerization by using piezoelectric measurements. I.Perrissin-Fabert, Y.Jayet, G.Merle, M.Feve. (à paraître dans J.Appl.Sc.) 